Archives de sciences sociales des religions

180 | octobre-décembre 2017

Bulletin bibliographique

\title{
Le Groupe de sociologie des religions du CNRS et les études sur l'islam
}

Constant Hamès

\section{OpenEdition \\ 12 Journals}

\section{Édition électronique}

URL : https://journals.openedition.org/assr/29720

DOI : $10.4000 /$ assr.29720

ISSN : $1777-5825$

Éditeur

Éditions de l'EHESS

\section{Édition imprimée}

Date de publication : 1 décembre 2017

Pagination : 69-76

ISSN : 0335-5985

\section{Référence électronique}

Constant Hamès, "Le Groupe de sociologie des religions du CNRS et les études sur l'islam », Archives de sciences sociales des religions [En ligne], 180 | octobre-décembre 2017, mis en ligne le 01 décembre 2019, consulté le 22 septembre 2021. URL : http://journals.openedition.org/assr/29720 ; DOI : https:// doi.org/10.4000/assr.29720 


\section{Constant Hamès}

\section{Le Groupe de sociologie des religions du CNRS et les études sur l'islam}

Je n'ai pas constitué de dossier sur l'histoire des études sur l'islam au sein du Groupe de sociologie des religions (G.S.R.) et encore moins sur l'histoire de cette équipe thématique du Centre d'études sociologiques du CNRS. Les lignes qui suivent ne doivent donc être comprises que dans le cadre d'un témoignage vécu, avec ses particularités sociologiques et psychologiques.

Pour situer chronologiquement ce vécu, je suis entré au G.S.R. à l'automne 1964, soit dix ans après sa création. Dans les faits, je ne suis pas entré directement au G.S.R. puisque j'ai été affecté, en tant que collaborateur technique, à un chercheur du Groupe, Jacques Maître, suivant la pratique du CNRS de l'époque. Ce statut de collaborateur attaché à un chercheur était l'expression du paysage social d'un CNRS fortement marqué par un esprit de hiérarchie ${ }^{1}$. Le G.S.R. était alors logé rue Cardinet, dans le $17^{\mathrm{e}}$ arrondissement de Paris mais je n'ai pas souvenir d'y avoir travaillé ni assisté à des réunions. Le travail se faisait chez moi et en réunion chez Jacques Maître, boulevard du Temple, dans le $3^{\mathrm{e}}$ arrondissement de Paris. Dans les faits, le Groupe, sur le plan du travail scientifique, fonctionnait plutôt comme une juxtaposition de chercheurs individuels que comme un vrai laboratoire de recherche. Mais il avait une extension, un bras armé scientifique sous la forme de la revue Archives de Sociologie des Religions dont le premier numéro semestriel paraît en 1956 et qui deviendra à partir de 1973 la présente Archives de sciences

1. Jacques Maître m'avait prévenu que l'administration du CNRS n'admettait pas à l'époque, dans les rapports d'activité, les publications à deux ou plusieurs auteurs. Cette disposition rendait de fait invisible le travail des collaborateurs techniques. Par exemple, dans mon réseau universitaire d'origine (j'avais terminé " psycho » à la Sorbonne et à l'Institut catholique en 1963), j'avais reçu le témoignage de Paul Fraisse (Fraisse, 1957) où, à la fois les expériences, leur analyse et la rédaction provenaient du travail de Nadine Zuili, collaboratrice technique. Ce fut, dans une bien moindre mesure, mon cas avec la parution par Jacques Maître de Les prêtres ruraux (Maître, 1967) où, à côté du dépouillement technique et statistique des questionnaires de l'enquête, j'avais rédigé une bonne partie des chapitres III et VII. Cette pratique disparut heureusement assez vite et, en 1966, Paul Fraisse et Nadine Zuili publiaient conjointement «L'estimation du temps » (Zuili, Fraisse, 1966) et en 1970, Jacques Maître associait Evelyne Aver, Constant Hamès et Guy Michelat à l'article «Pratique religieuse et comportement électoral à travers les sondages d'opinion " (Aver, Hamès, Maître, Michelat, 1970). 
sociales des religions. Et c'est bien là que la dynamique "sociologie des religions" était à l'œuvre. Faute d'avoir dans ses rangs des chercheurs travaillant sur un éventail élargi de religions, la revue suppléait à ce manque à travers ses deux rubriques, les articles eux-mêmes et ce qui a longtemps été considéré comme son fleuron éditorial, le «Bulletin bibliographique», sur lequel Émile Poulat régnait alors ${ }^{2}$.

En 1964-1965, au moment de mon entrée, le G.S.R., en tant que tel, était christiano-centré, avec un fort contingent d'enquêtes sur le catholicisme hexagonal, hormis Henri Desroche, aux horizons plus «tiers-mondistes». Certains de ses chercheurs avaient quitté la prêtrise ou les ordres religieux, ce qui était le cas de Poulat et de Desroche, parmi les fondateurs, puis de Jean Séguy ${ }^{3}$ et de JeanPierre Deconchy. Serge Bonnet, membre un peu fantôme exilé en Lorraine, et René Luneau, venu plus tard, appartenaient tous deux à l'ordre dominicain.

Sans surprise, l'islam n'était pas à l'ordre du jour dans ce cénacle. Celui qui aurait pu s'en approcher quelque fois était Desroche, dans ses recherches sur les mouvements coopératifs plus ou moins messianiques, mais son échantillon restait à l'intérieur de la mouvance chrétienne, même internationale. Une exception me revient en mémoire. C'est en suivant un de ses séminaires (très chaleureux), avenue Franco-russe à Paris, que j'ai découvert un étudiant sénégalais préparant une thèse sur le mouvement coopératif musulman des Mourides, au Sénégal. La thèse, parue en livre en 1969 (Tidjane Sy, 1969), relatait le passage au collège coopératif de la $6^{\mathrm{e}}$ section de l'EPHE et l'apport du séminaire de Desroche:

Nous n'avons nullement le droit de le nier: ce qui nous tue souvent, ce sont nos propres déterminismes et n'eut été, en ce qui me concerne, la rigueur et l'intransigeance intellectuelle du professeur Henri Desroche qui m'a initié à la recherche, j'aurais abordé ce travail avec l'idée que les Mourides n'étaient rien d'autre que des hérétiques. Qu'Henri Desroche soit remercié, ici, pour m'avoir guidé, avec sa vigilance habituelle (ibid.: 9).

La remarque prend du relief lorsqu'on sait que l'auteur, Cheikh Tidjane Sy, appartenait comme son nom l'indiquait à la confrérie «concurrente» de celle des Mourides, la Tijâniyya. Cette exception étant, lorsqu'on parcourt l'œuvre d'époque de Desroche et, par exemple, ses Sociologies religieuses (Desroche, 1968), le mot islam n'y apparaît qu'une seule fois, dans une note de bas (ibidem: 19). Certains membres du Groupe avaient cependant eu, d'une façon ou d'une autre, des contacts avec des populations musulmanes. Séguy, dont les parents avaient vécu en Algérie et lui-même ayant travaillé sur les moines coptes au Caire, s'est révélé être le plus attentif à mes démarches de recherche sur les Africains musulmans immigrés. Maître avait connu la Turquie dans une partie de son enfance et Deconchy qui avait enseigné au Liban, me récitait, dans les années 1960, les chiffres en arabe...

Lorsque je suis devenu collaborateur technique de Maître, je ne m'intéressais pas à l'islam dont je n'avais d'ailleurs aucune idée. La Belgique, dont j'étais originaire, ne possédait pas en effet de colonies musulmanes et donc pas de

2. Lors de mes premiers comptes rendus bibliographiques, une ou deux relectures avec corrections étaient faites par Émile Poulat, en une forme de rite de passage ou d'initiation, sur le modèle scolaire. 3. Jean Séguy avait gardé une sorte de «capital incorporé » de son ancien état de postulant (malheureux) de la Compagnie de Jésus et un jour où il m'avait invité dans une pizzeria près de chez lui, le serveur, prenant la commande, lui demande : "Et vous Monsieur l'Abbé ?". 
recherche orientaliste. J'étais plutôt à la découverte de la sociologie et de ses méthodes, perspective d'analyse que je voulais ajouter à celle de la psychologie. D'où l'attelage avec Maître où le fond catholique des investigations importait moins que les méthodes d'enquête et de calcul. Ma solide formation statistique en psychologie (au-delà du très en vogue $k h i-d e u x^{4}$ ) s'alliait bien avec les modèles mathématiques de Maître. Mais nous parlions aussi beaucoup, entre nous, de psychologie sociale et clinique et, plus tard, d'ethnologie; la suite du parcours de Maître (psychanalyse sociohistorique, ethnographie vietnamienne) fait écho à bien des discussions de l'époque.

Ce n'est qu'après 1965, à la suite d'un séjour inattendu mais décisif parmi les nomades (musulmans) du désert mauritanien, que j'ai commencé à me documenter sur la culture et la langue arabe de cette population et, forcément, sur sa religion, quoiqu'elle ne me soit jamais apparue, en ces années-là, en avant-plan du fonctionnement et des enjeux de la société. À partir de ce moment, je me suis senti doublement en décalage au sein du G.S.R. Non seulement je ne pouvais pas y trouver d'appui pour me former aux études sur l'islam ou simplement échanger sur le sujet mais au surplus mon islam en question se situait en Afrique. Et étudier les populations africaines relevait alors de l'ethnologie et non pas de la sociologie ( «Eux et nous»!). Institutionnellement, au CNRS, ces deux «disciplines » relevaient de commissions différentes et étanches ${ }^{5}$. L'appartenance à un laboratoire de sociologie entraînait des conséquences pratiques et financières, comme l'absence de crédits de mission pour aller travailler sur «le terrain» en Afrique ${ }^{6}$. Faut-il ajouter une difficulté supplémentaire qui a perduré jusqu'à tout récemment: l'ethnologie ne comportait pas de recherches sur l'islam africain et ceux qui auraient pu en mener, les «orientalistes ", à une ou deux rares exceptions près, ne regardaient que du côté du Maghreb ou de l'Orient. L'histoire de Georges Balandier m'avait alors parue révélatrice. Il avait choisi comme premier terrain d'études la Mauritanie, avec Paul Mercier, au début des années 1950. Il tente une étude sur les griots et leur littérature mais s'avoue rapidement vaincu par les obstacles, dont le principal était la langue arabe et abandonne ses recherches pour partir au Gabon ${ }^{7}$.

Ma planche de salut fut alors le «Bulletin bibliographique» des Archives. C'est à travers tous les livres commandés ou reçus et leurs comptes rendus que j'ai petit à petit découvert l'immensité du savoir à enregistrer pour faire une sociologie

4. Lors de la soutenance de thèse de Raymond Boudon (L'analyse mathématique des faits sociaux, Sorbonne, 1967), au contenu moitié mathématique moitié sociologique (comme la composition du jury), Jean Stoetzel, son directeur de thèse, incommodé par la publication de la thèse avant qu'elle ne soit soutenue, s'indignait de ne pas y trouver le moindre khi-deux ! Ma première publication (Hamès, Marcus-Steiff, 1967), dont le titre prête à sourire, présentait des applications statistiques pour l'ordinateur de bureau CAB 500 de Bull, installé au Centre d'études sociologiques.

5. L'histoire et la sociologie de la construction académique des disciplines au CNRS mériterait son lot d'études.

6. Cette impossibilité d'aller sur le terrain africain explique qu'après mai 1968 et jusqu'en 1970, après ma démission du CNRS, je sois parti travailler au Sahara algérien puis mauritanien puis à nouveau en Algérie (Aurès) dans le cadre de sociétés privées, dans le seul but de pouvoir accéder à des terrains d'études et d'y progresser en langue arabe. Mon retour au CNRS ne s'est fait, par «la petite porte», qu'en 1975 .

7. Georges Balandier n'avait pas oublié la Mauritanie en acceptant de publier, en 1969, mon premier article qui portait sur cette société, dans ses Cahiers Internationaux de Sociologie. 
ou une ethnologie de l'islam. Ma formation aux pensées et pratiques islamiques s'est ainsi faite à travers la bibliographie de la revue puis à travers les cours de langue et de civilisation arabes que je suivais à l'École Nationale des Langues Orientales Vivantes, vers la fin des années $1960^{8}$.

En parcourant la rubrique «Bulletin des périodiques et des ouvrages », on constate en effet qu'à partir du premier numéro de 1956, des titres de quelques études sur l'islam sont recensés et la question est ainsi évoquée par FrançoisAndré Isambert: «Grâce aux soins diligents d'une de nos collaboratrices, nous nous trouvâmes bientôt à la tête de 600 et quelques titres concernant l'Islam. En un sens, tout est religieux dans l'Islam. Et pourtant, nombre de ces études éveillaient en nous des résonances avant tout politiques, juridiques ou démographiques. Bref, par une opération sans doute illégitime, nous étions obligés d'utiliser ici des concepts émanant d'autres formes de civilisation, pour opérer un tri dont la précarité nous paraît trop évidente". Ce même premier numéro recensait "les thèses et mémoires de 1940 à 1954 ", dont 18 titres sur l'islam. On trouve ainsi, dans les recensions de la revue, au fil des ans, les noms de Jacques Berque, Joseph Chelhod, Gustave E. Von Grunebaum, Tor Andrae, Louis Massignon, René Brunel, etc. Mes premiers comptes rendus sur des ouvrages relatifs à l'islam datent de 1965, à côté de semblables et premières contributions de Jean Paul Charnay. Ce "pas de deux » a continué jusqu'à la cessation des relations de Charnay avec le Groupe, vers 1974. Après un entracte épisodique entre 1968 et 1973, j'ai continué à fournir à la revue des comptes rendus jusqu'aux débuts des années 2010.

Parmi les articles propres publiés par la revue, dans ses débuts (années 19501960), l'islam est présent mais de façon mineure et sporadique. Le premier article publié fut celui de Berque portant sur «Quelques problèmes de l'islam maghrébin» (Berque, 1957), dans lequel «quelques » veut plutôt dire "principaux ». Sans entrer dans le détail et l'analyse des articles divers publiés, on remarquera le $\mathrm{n}^{\circ} 15$ de 1963 qui comprend trois contributions portant sur le thème du développement au Pakistan (Eister, 1963), en Iran (Jacobs, 1963) et dans le Sud-est asiatique (Saran, 1963), accompagné d'un article de Maxime Rodinson sur les travaux de W. Montgomery Watt (Rodinson, 1963), dont il relevait le caractère sociologique. De même, on notera la série de trois articles originaux fournis par Vincent Monteil sur «Une confrérie musulmane: les Mourides du Sénégal » (Monteil, 1962), «Lat Dior, Damel du Kayor et l'islamisation des Wolofs » $\left(\mathrm{n}^{\circ} 16,1963\right)$ et «Un visionnaire musulman sénégalais » (n 19, 1965). À noter encore l'article de Vittorio Lanternari sur "Les Black Muslims, du messianisme populaire à l'institution bourgeoise" (Lanternari, 1967) et celui de René Luc Moreau sur le «hamallisme» en Haute Volta dans «Les marabouts de Dori» (Moreau, 1964). On constate d'ailleurs que l'islam africain est le mieux représenté dans ces publications.

8. Influencé néanmoins par les travaux du G.S.R., notamment par la question du dénombrement des pratiquants catholiques qui était un de ses axes premiers, j'avais voulu tenter quelque chose dans ce sens sur l'islam des Africains en France. Habitant à côté de la mosquée de Paris, j'avais remarqué que le seul signe de vie qui s'y manifestait était représenté par les quelques fidèles dont des Africains qui entraient et sortaient par la petite porte pour la prière du vendredi. En 1965, j'avais écrit au directeur pour m'autoriser à pénétrer dans la mosquée et faire des relevés des musulmans présents à la prière du vendredi. La réponse avait été cinglante: l'islam est une question de foi et n'a rien à voir avec des dénombrements et des comptages! 
À partir de l'année 1968 et jusqu'au début des années 1980 (Khomeini arrive au pouvoir en 1979), un grand vide s'est installé dans les publications de la revue sur l'islam, correspondant aux dernières années de l'époque de la «sécularisation ». Un article de Charnay (voir plus loin) paru en 1974 y fait exception. En 1969, je crois, j'ai présenté pour la première fois un projet de recherche au CNRS portant sur Islam et immigration, sans rencontrer d'écho9. Poulat m'avait conseillé d'ajouter «culture» dans le titre. L'énorme sociologie de l'immigration travaillait en effet dans une atmosphère complètement "sécularisée ", sur des voyageurs "sans bagages ". Mon premier article sur l'islam dans les Archives est publié en 1980 (Hamès, 1980) et intègre une problématique nouvelle qui se fait jour dans le monde musulman et qui n'aura de cesse de prendre de l'ampleur dans les décennies suivantes, sous des étiquettes changeantes: "Deux aspects du fondamentalisme islamique. Sa signification au Mali actuel et chez Ibn Taymîya ». Et en 1982 (n 55-1) paraît le premier d'une série de numéros thématiques sur l'islam: "Personnels et groupes religieux en islam ", avec Yann Richard, Marc Gaborieau, Sossie Andezian, Constant Hamès et Jean-François Clément. En 1989, paraissent deux numéros (n 68-1 et 2) consacrés à "L'islam en Europe", avec Anne-Marie Brisebarre, Chantal Saint Blancat, Liliane Kuczynski, Altan Gokalp, Sossie Andezian et Constant Hamès. Le n ${ }^{\circ}$ 68-2 comportait une bibliographie d'environ 250 titres (époque sans internet!). La situation, à la fois institutionnelle et sociologique, des Archives avait changé, dans un contexte intérieur et extérieur plus ouvert. Son ouverture multidisciplinaire et multiculturelle s'affirme nettement.

Avant les années 1980, le problème majeur, que je vivais directement, en ce qui concerne les recherches sur l'islam au sein du G.S.R. était la question classique du recrutement. J'ai tenté vainement durant les années 1970 de faire venir au moins un collègue dans le Groupe, pour créer une équipe de recherche sur l'islam. Il faut avouer que mon statut de technicien ITA, que j’ai gardé jusqu'en 1983, ne facilitait pas mes démarches, non plus que les finances du Groupe, toujours aussi exigües. Je suis donc resté seul représentant de la recherche sur l'islam au G.S.R. jusqu'à sa scission en deux laboratoires distincts en 1993-1995 ${ }^{10}$.

Le Groupe a sans doute eu conscience de ce manque puisqu'au cours des années 1960 il a noué une sorte de partenariat avec Jean-Paul Charnay, islamologue, sans que celui-ci n'intègre la structure. "Pied noir ", il était juriste de formation et non sociologue ou ethnologue. Son énorme thèse portait sur les scrutins politiques en France depuis 1815. Il s'était fait connaître par un ouvrage sur la jurisprudence musulmane en Algérie (Charnay, 1965) et par une collaboration avec Jacques Berque. Notons que l'ouvrage collectif qu'ils firent paraître en 1966 (Berque, Charnay, 1966) réserve une surprise; parmi les contributeurs, en dehors d'eux-mêmes, l'on trouve Henri Corbin, Mohamed Hamidullah et... Gabriel Le Bras. Ce dernier avait en effet noué des relations avec Berque et avait participé à des débats d'où l'ouvrage est sorti. Les éditeurs ont reproduit sur

9. J'avais publié un premier article sur le sujet: Hamès, 1979.

10. Le Centre d'études interdisciplinaires des faits religieux (CEIFR) du côté de l'EHESS (1993), sous la direction de Danièle Hervieu-Léger, et le Groupe Sociologie, Religions, Laïcités (GSRL) du côté de l'EPHE, sous la direction de Jean Baubérot. 
trois pages et demie le schéma de son intervention orale dans ces débats, schéma qui s'intitulait "Perspectives de recherche en matière de sociologie religieuse ».

De son côté, Charnay publie en 1977 Sociologie religieuse de l'Islam (Charnay, 1977) dont il présente l'argumentaire dans un article de 1974: «Préalables épistémologiques à une sociologie religieuse de l'Islam» (Charnay, 1974). Sans entrer dans le maquis d'abstractions qui tapisse son ouvrage et son article, on peut dire qu'à ses yeux la sociologie telle qu'elle existait ne pouvait pas être appliquée à l'islam. Il proposait donc non pas «la constitution d'une sociologie religieuse de l'islam mais la définition de prolégomènes méthodologiques destinés à être dépassés, dans la mesure où ils permettront d'œuvrer avec moins d'incertitude et plus de réalité psychologique et sociologique islamique à la construction de techniques spécifiques, à partir desquelles les véritables études de sociologie religieuse musulmane pourraient enfin démarrer» (ibid.: 86). Vision essentialiste de l'islam ou une anecdote sur les contours de son épistémologie ? En 1967-1968, j'écris un premier article, après lecture de Homo hierarchicus de Louis Dumont, que j'intitule «La société maure ou le système des castes hors de l'Inde » (Hamès, 1969), avec l'idée de mettre en cause, par un exemple ethnographique, l'usage strictement monographique d'un concept, celui de caste. Le Groupe m'envoie consulter Charnay qui me reçoit dans un petit local rue Monsieur-le-Prince. Il se demande «comment on peut comparer quelques pauvres nomades courant derrière leurs chameaux à un immense sous-continent comme l'Inde? ». Et il me donne un excellent conseil: "Apprenez l'arabe». Je n'ai compris que plus tard que ce conseil résonnait sans doute chez lui comme une sorte de regret, lui qui n'avait jamais "appris l'arabe ». Ayant alors rompu avec le Groupe, suite, semble-t-il selon certaines rumeurs, à des désaccords d'ordre politique, il a par la suite dirigé à la Sorbonne un Centre de philosophie de la stratégie. J'ai d'ailleurs participé à une séance de ce centre, au début des années 1970, consacrée à la situation de thèse d'un de mes amis tunisiens, portant sur la question des frontières entre la Tunisie et la Libye. Je me suis aperçu que la majorité des auditeurs étaient des officiers de l'armée. J'ai eu un dernier et bref contact avec lui à Bamako (Mali) en janvier 1979. Revenant de deux mois de recherche dans la «brousse » malienne, dans la région de Niorodu-Sahel, fief des adeptes de Shaykh Hamallah, j'allais faire valider mon billet de retour vers la France dans une agence du centre de Bamako et j'ai la surprise de me trouver face à Charnay. Il tenait en mains une impressionnante liasse de billets d'avion à la destination de nombreuses capitales africaines. Il était resté deux jours à Bamako et sans doute en a-t-il été de même dans les autres capitales. Il menait une enquête sur l'islam en Afrique et ses informations provenaient des institutions françaises telles que les ambassades. L'année suivante, il publiait «Islam et négritude. Réflexions sur l'Afrique occidentale» (Charnay, 1980).

Le Groupe a par la suite entretenu pendant quelque temps des relations fructueuses avec Mohammed Arkoun. Sa pensée et sa personnalité généreuse m’ont marqué (Hamès, 1985). Je lui dois la publication dans sa revue Arabica, de mon premier article sur la magie en islam, alors qu'à l'époque le sujet prêtait plutôt à sourire (Hamès, 1987). Et la sociologie, dans tout ceci ? La remarque de Berque, dans l'ouvrage collectif Normes et valeurs, résume bien ce qui se passait en France et ailleurs dans les années 1950-1960: 
Le sociologue orientaliste doit sortir du ghetto où il s'est longtemps confiné. C'est grande pitié de voir à quel point cette province de nos études a peu fourni à des disciplines générales comme l'anthropologie et la sociologie et combien peu elle s'en est inspirée (Berque, Charany: 101).

Berque lui-même s'est souvent laissé enchanter par le modèle de la littérature arabe ou islamique sur laquelle il travaillait, en faisant de la langue le lieu privilégié où chercher la réponse aux questions soulevées ${ }^{11}$.

Pour conclure sur ces années 1950-1980, sans en être absent l'islam n'était pas l'objet d'une attention particulière au sein du G.S.R. C'est dans les pages de la revue Archives que l'on en retrouve le plus de traces. L'islam d'alors était le plus souvent un islam d'ailleurs et un islam non revendicatif ou idéologisé. Son intrusion en France et en Europe et sur la scène internationale, sous forme d'actualité sociale et politique, à partir des années 1980, a provoqué un réveil brutal et un redéploiement de la recherche face aux données nouvelles.

Constant HamÈs

Centre d'études en sciences sociales du religieux (CéSor, CNRS-EHESS) constant.hames@ehess.fr

\section{Bibliographie}

Aver Evelyne, Hamès Constant, Maître Jacques, Michelat Guy, 1970, «Pratique religieuse et comportement électoral à travers les sondages d'opinion ", Archives de sociologie des religions, 29-1, p. 27-52.

BERQUE Jacques, 1957, "Quelques problèmes de l'islam maghrébin », Archives de sociologie des religions, 3, p. 3-19.

Berque Jacques, Charnay Jean-Paul (éds.), 1966, Normes et valeurs dans l'islam contemporain, Paris, Payot.

Charnay Jean-Paul, 1965, La vie musulmane en Algérie d'après la jurisprudence de la première moitié $d u \mathrm{xx}^{e}$ siècle, préface de Jacques Berque, Paris, Presses universitaires de France.

-, 1974, «Préalables épistémologiques à une sociologie religieuse de l'Islam », Archives de sciences sociales des religions, 37 , p. 79-86.

-, 1977, Sociologie religieuse de l'Islam, Paris, Sindbad.

-, 1980, « Islam et négritude. Quelques réflexions sur l'Afrique occidentale », L'Afrique et l'Asie Modernes, 126-2, p. 3-16.

Desroche Henri, 1968, Sociologies religieuses, Paris, Presses universitaires de France, coll. «Le sociologues».

EISTER Allan W., 1963, «Perspectives sur les fonctions de la religion dans un pays en voie de développement: l'Islam au Pakistan ", Archives de sociologie des religions, 15, p. 35-42.

Fraisse Paul, 1957, Psychologie du temps, Paris, Presses universitaires de France.

Hamès Constant, 1969, «La société maure ou le système des castes hors de l'Inde ", Cahiers Internationaux de Sociologie, 46, p. 163-177.

11. Sur cette question, voir aussi Hamès, 1999. Une erreur à corriger, p. 178: Joseph Chelhod n’est pas «juif syrien » mais «chrétien syrien». 
-, 1979, «Islam et structures sociales chez les immigrés Soninké en France », Social Compass, 36-1, p. 87-98.

-, 1980, «Deux aspects du fondamentalisme islamique. Sa signification au Mali actuel et chez Ibn Taymîya ", Archives de sciences sociales des religions, 50-2, p. 177-190.

-, 1985, "Mohammed Arkoun et la pensée islamique: pour un sursaut ", Archives de sciences sociales des religions, 60-2, p. 187-193.

-, 1987, «Taktub ou la magie de l'écriture islamique. Textes soninké à usage magique", Arabica, XXXIV, 3, p. 305-325, 15 illustrations.

-, 1999, «Islam et sociologie: une rencontre qui n'a pas eu lieu ?» in Voyé L. et Biliet J. (éds.), Sociologie et religions. Des relations ambigües, Leuven, Leuven University Press, coll. «Kadoc-Studies», p. 171-182.

Hamès Constant, Marcus-STEIFF Joachim, 1967, «Quelques programmes statistiques en langage PAF », Revue française de sociologie, 8-1, p. 68-71.

Jacobs Norman, 1963, "La religion et le développement économique: le cas de l'Iran ", Archives de sociologie des religions, 15, p. 43-48.

LANTERNARI Vittorio, 1967, «Les Black Muslims, du messianisme populaire à l'institution bourgeoise ", Archives de sociologie des religions, 24, p. 105-120.

MAîTRE Jacques, 1967, Les prêtres ruraux, Paris, Éditions du Centurion, coll. «Études de sociologie».

MonTeil Vincent, 1962, «Une confrérie musulmane: les Mourides du Sénégal », Archives de sociologie des religions, 14, p. 77-102.

-, 1963, "Lat Dior, Damel du Kayor et l'islamisation des Wolofs» Archives de sociologie des religions, 16, p. 77-104.

-, 1965, «Un visionnaire musulman sénégalais », Archives de sociologie des religions, 19, p. 69-98.

Moreau René Luc, 1964, «Les marabouts de Dori» Archives de sociologie des religions, 17 , p. 113-134.

Rodinson Maxime, 1963, "Une étude sur l'islam ", Archives de sociologie des religions, 15, p. 137-143.

SARAN A. K., 1963, «Hinduism and economic Development in India ", Archives de sociologie des religions, 15, p. 87-94.

Tidjane Sy Cheikh, 1969, La confrérie sénégalaise des Mourides. Un essai sur l'Islam au Sénégal, Paris, Présence Africaine.

Zuili Nadine, Fraisse Paul, 1966, "L'estimation du temps en fonction de la quantité de mouvements effectués dans une tâche. Étude génétique ", L'Année psychologique, 66-2, p. 383-396. 